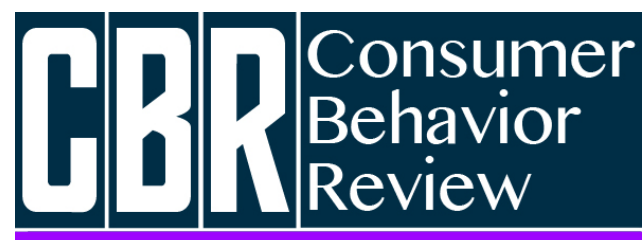

Revista Comportamento do Consumidor
Aquino, S. D., Amigo, A., \& Zehoul, A. (2021). "Então é Natal, e o que você fez?" Um estudo sobre o impacto das ativações promocionais na compra de panetones. Consumer Behavior Review, 5(1), 45-59.
ISSN: 2526-7884

Editor: Prof. Dr. Marconi Freitas da Costa

E-mail: cbr@ufpe.br
Avaliação: Double blind review

Recebido: 11 de Junho, 2020

Aceito: 15 de dezembro, 2020

\title{
“ENTÃO É NATAL, E O QUE VOCÊ FEZ?” UM ESTUDO SOBRE O IMPACTO DAS ATIVAÇÕES PROMOCIONAIS NA COMPRA DE PANETONES
}

\author{
"And so this is Christmas, and what have you done?" A study on the impact of promotional \\ activations on the purchase of panettones
}

\author{
Sibele Dias de Aquino ${ }^{1}$ \\ ORCID: https://orcid.org/0000-0003-1391-0911 \\ E-mail: sibele.aquino@gmail.com \\ Alice Amigo ${ }^{1}$ \\ ORCID: http://orcid.org/0000-0003-3567-1691 \\ E-mail: aliceamigo@yahoo.com.br \\ Anne Zehoul ${ }^{1}$ \\ ORCID: http://orcid.org/0000-0002-6580-761X \\ E-mail: annezehoul@yahoo.com.br
}

\section{${ }^{1}$ Pontifícia Universidade Católica do Rio de Janeiro, Rio de Janeiro, Brasil}

\begin{abstract}
Resumo
Em um contexto comercial amplamente diverso, compreender como as ativações promocionais no varejo impactam as decisões de compra dos consumidores é um desafio cada vez maior. 0 objetivo deste estudo foi verificar se ativações promocionais de produtos sazonais estimulam o consumidor a comprar itens sem planejamento, durante o final do ano. Para isso, foram coletados dados de 131 adultos brasileiros, sendo 48,9\% homens e $51,1 \%$ mulheres, com média de idade de 44,2 anos (DP $=10,88)$. Os
\end{abstract}

\begin{abstract}
In a widely diverse commercial context, understanding how promotional activations in retail impact consumers' purchasing decisions is a growing challenge. In this study, our goal is to verify if promotional activations of seasonal products stimulate the consumer to buy items without planning during the holiday season. For this, we collected data from 131 Brazilian adults, $48.9 \%$ male, and $51.1 \%$ female, with an average age of 44.2 years $(S D=10.88)$. The respondents filled out an online questionnaire that contained
\end{abstract}


respondentes preencheram um questionário de autorrelato online que continha diversas questões sobre dados sociodemográficos, hábitos e preferências de compra, além de uma escala psicométrica de tendência a comprar impulsivamente. Esses dados comportamentais foram analisados quantitativamente por meio de software estatístico SPSS e os resultados indicam que o planejamento individual pode ter maior efeito sobre a decisão de compra de produtos sazonais do que algumas ativações promocionais. Considerando os produtos testados (chocotones e panetones), a suscetibilidade a comprar por impulso pode ser determinada por outros fatores, além dos estímulos ambientais. Discute-se a caracterização da amostra, a consistência com estudos anteriores e algumas limitações do estudo. Conclui-se que futuras abordagens podem abordar importantes variações interdisciplinares para aprofundamento do tema.

Palavras-chave: Ativação Promocional; Produtos Sazonais; Compra por Impulso. several questions about sociodemographic data, habit and purchasing preferences, besides a psychometric scale of impulse buying tendency. The data were analyzed using SPSS statistical software, and the results show that individual planning can have a greater effect on the decision to purchase seasonal products than some promotional activations. Considering the products assessed (chocotones and panettones), the susceptibility to impulse buying can be determined by other factors than environmental stimuli. The characterization of the sample, the consistency with previous studies and some limitations of the study are discussed. We conclude that, to deepen our understanding about seasonal products, future approaches may address important interdisciplinary variations.

Keywords: Promotional Activation; Seasonal Products; Impulse Buying.

This work is licensed under a Creative Commons Attribution 4.0 International License.

\section{INTRODUÇÃO}

Atualmente, capturar a atenção dos clientes tem se tornado um desafio no varejo brasileiro: segundo estudo do Instituto Nielsen Media Research (2015), 70\% das decisões de compra acontecem na loja. Mesmo quando já tem planejadas as categorias de produto que pretende comprar, o consumidor prefere decidir nas lojas qual marca escolher, e, em média, gasta apenas 15 segundos diante de uma gôndola (Nielsen, 2017). 0 Point of Purchase Advertising International (POPAI) relata que $76 \%$ das compras em supermercados não são planejadas. Diante deste contexto, é cada vez mais importante que tanto as indústrias quanto o varejo compreendam os fatores que mais impactam a escolha dos consumidores quando estão comprando nas lojas. Tais fatores podem ser intrínsecos, relacionados a motivações e tendências individuais, ou extrínsecos, relacionados a influências do ambiente.

Para Kotler (1973), a atmosfera do local em que a compra é feita é mais influente na decisão de compra do que o produto em si, sendo uma ferramenta de marketing relevante para os varejistas. A música, o odor, as pessoas, os ruídos, a configuração espacial, a exposição dos produtos e outros elementos na loja podem exercer influência sobre o comportamento de compra. Diversas táticas são utilizadas por indústrias e varejistas para promoção de produtos nas lojas, tais como uso de sinalizações, anúncios, diferentes localizações e espaços concedidos aos produtos.

Essa disputa pela atenção do consumidor se torna ainda mais relevante em datas comemorativas, principalmente no Natal, que é a principal data comemorativa do varejo brasileiro e movimentou cerca de $\mathrm{R} \$ 35,9$ milhões em 2019 (Valor, 2019). O presente estudo faz, portanto, um recorte nesse período. Mais especificamente, o objetivo principal da pesquisa é verificar se ativações promocionais de produtos sazonais estimulam o consumidor a comprar itens sem planejamento, durante o final do ano. Assim, foi explorado o período natalino e as ativações promocionais da 
categoria de panetones e chocotones nas lojas de varejo para testar o quanto estimulam o consumidor a comprar esses produtos por impulso. Presente em 53,6\% dos lares brasileiros, o panetone é um símbolo do Natal e alcançou R 739 milhões em vendas no ano de 2019 (Silveira, 2019).

Há uma vasta literatura acadêmica que investiga os impactos das tendências individuais e dos efeitos ambientais no comportamento de compra do consumidor e em seu potencial de gerar compra por impulso (Bailey \& Areni, 2006; Bawa, Landwehr, \& Krishna, 1989; Sevilla \& Townsend, 2016; Singh et al., 2014). Khachatryan et al. (2018) foram os primeiros a abordar como a impulsividade de compra se relaciona com fixações visuais em informações de ponto de venda. Os autores propuseram que a inclusão de produtos potencialmente comprados por impulso (por exemplo, doces) com uma quantidade crescente de mensagens de marketing de ponto de venda poderia esclarecer as relações entre tipo de produto, atenção visual e compra impulsiva (Khachatryan et al., 2018).

Entretanto, não foram encontrados estudos com foco na categoria de produtos sazonais, de oferta esporádica, ainda que esses itens tenham grande relevância, tanto para o consumidor quanto para o varejo, durante datas comemorativas importantes no calendário varejista brasileiro. Tais itens sazonais têm características específicas, como incerteza de disponibilidade futura e de utilidade ao longo do tempo (Soysal \& Krishnamurthi, 2012), que influenciam as respostas do consumidor a diferentes estímulos de marketing. 0 presente estudo, assim, pretende oferecer subsídios para ampliar o entendimento sobre o comportamento do consumidor na compra de produtos sazonais enquanto está na loja física.

Utilizando-se de um estudo descritivo e correlacional com dados coletados por meio de um questionário online, esta pesquisa traz achados que podem impactar significativamente as decisões de investimento e as estratégias de vendas de panetones e chocotones, tanto para varejistas quanto para a indústria. A principal conclusão deste estudo é que o planejamento prévio de compra parece ser mais relevante para a amostra analisada do que qualquer tipo de ativação em loja ou motivação espontânea. A lembrança de ter visto ativações promocionais de panetones e chocotones não aumentou a quantidade de compra dos que já tinham planejado, e tampouco motivou os indivíduos que não tinham planejado a comprarem.

Este artigo está estruturado em seis seções. Seguindo a introdução, a segunda seção apresenta uma breve revisão de literatura dividida em subseções. A terceira seção expõe o método utilizado e a quarta seção apresenta os resultados. A quinta seção discute os principais achados, jogando luz sobre as particularidades do que foi apresentado e considerando as limitações do estudo. Por último, a sexta seção apresenta implicações gerenciais e recomendação de estudos futuros.

\section{REFERENCIAL TEÓRICO}

\section{Motivações de Compra que Impactam o Comportamento de Compra por Impulso}

A compra por impulso é definida como uma "tendência do consumidor para comprar espontaneamente, sem reflexão, de forma imediata, estimulado pela proximidade física do objeto desejado, dominado pela atração emocional e absorvido pela promessa de gratificação imediata" (Rook \& Fisher, 1995, p. 306). Sendo mais da metade das decisões de compra no varejo não são planejadas (Ståhlberg \& Maila, 2010), o papel que a impulsividade de compra desempenha no ponto de venda pode ser predito por motivações intrínsecas, como o hedonismo, e por aspectos extrínsecos, como o ambiente físico de compra.

\section{Motivações Individuais de Compra: Aspectos Hedônicos e Utilitários}

0 consumo hedônico pode ser definido como a faceta do comportamento de compra que se relaciona a aspectos de fantasia, emotivos e multissensoriais do consumo (Hirschman \& Holbrook, 1982). Segundo Arnold e Reynolds (2003), os motivos do consumo hedônico são parecidos com a orientação para tarefa do consumo utilitário, sendo que a diferença está na tarefa, que neste caso está relacionada à realização hedônica, como experimentar diversão, fantasia e estímulo sensorial.

Segundo Chang e Fang (2012), motivações utilitárias e hedônicas não são excludentes. Pode haver pessoas que percebem apenas um desses dois aspectos e outras que percebem ambos os aspectos ao mesmo tempo enquanto realizam compras em varejos de diferentes formatos. Estudo de 
Yim et al. (2013) revelou que as motivações hedônicas em uma loja de supermercado (onde se vendem primariamente produtos utilitários, para atender a necessidades consideradas básicas) são similares a de um shopping center (onde se oferece maior quantidade de produtos hedônicos, voltados à estimulação sensorial e gratificação). Independente do ambiente, quanto maior era motivação hedônica, maior a tendência a comprar por impulso e, consequentemente, maior o volume comprado na loja (Yim et al, 2013).

Arnold e Reynolds (2003) identificaram seis categorias de motivações hedônicas: (1) motivação de aventura, (2) motivação de socialização, (3) motivação de gratificação, (4) motivação de ideia (procura por novos produtos, inovações), (5) motivação de comprar para outras pessoas (role shopping) e (6) motivação de busca de valor (buscar descontos, liquidações etc.). Alguns estudos mostram que as motivações de consumo hedônicas atuam como antecedentes da impulsividade do consumidor, o que por sua vez levam à compra imediata (Beatty \& Ferrell, 1998; Bhardwaj \& Manchiraju, 2017; Bloch et al. 1986; Hirschman \& Holbrook, 1982).

Dentre as seis motivações hedônicas, o estudo Cinjarevic et al. (2011), utilizando-se da categoria de vestuário, apontou que as motivações de aventura, gratificação, valor e ideia estão mais associadas à compra por impulso enquanto as motivações de role shopping e socialização não tiveram relação significativa com este comportamento. No caso do role shopping uma possível explicação, segundo os autores, pode ser o fato de que ao buscar um produto para outras pessoas (um presente para amigos ou parentes), a compra adquire aspecto de tarefa, em que o valor utilitário passa a ser maior do que o valor hedônico. Uma conclusão parecida está no estudo sobre comportamento de compra de presentes de Natal de Fischer e Arnold (1990): as compradoras mulheres, principalmente, consideram as compras de Natal como uma tarefa importante, um trabalho sério a ser realizado.

Relacionando esses achados à categoria de panetones e chocotones, depreende-se que estes produtos podem ter motivações de compra distintas (Laroche et al, 2000) que, por sua vez, podem impactar de maneiras distintas o comportamento por impulso. Se a compra de panetones e chocotones for considerada pelo consumidor como uma tarefa associada diretamente aos afazeres e responsabilidades do período que envolve os festejos de Natal, pode haver menor ou maior tendência à compra por impulso, de acordo com a motivação hedônica dos indivíduos. Neste caso, torna-se relevante uma investigação de como os efeitos extrínsecos, ligados ao ambiente da compra, impactam o comportamento de compra por impulso deste item.

\section{Efeitos Ambientais no Comportamento do Consumidor no Ambiente Físico}

Kotler (1973) cunhou o termo atmosférico para descrever "o desenho consciente do espaço para criar certos efeitos no comprador". Essa variável ambiental pode ser considerada um dos estímulos que levam a algum efeito cognitivo no consumidor, influenciando respostas comportamentais (Turley \& Milliman, 2000). Sendo a compra planejada ou não, estímulos na loja levam à tomada de decisões durante o processo de compra (Inman, Winer, \& Ferraro, 2009).

Pesquisas clássicas manipularam elementos no ambiente de compra para analisar os efeitos de resposta no comportamento de consumo (Cox, 1970; Frank \& Massy, 1970; Kotzan \& Evanson, 1969; Smith \& Curnow, 1966). Outros estudos investigaram de que maneira o comportamento dentro do ambiente de loja é afetado pela influência de diferentes estímulos, incluindo variáveis como tempo despendido e percepções do consumidor. Dentre esses estudos, destacam-se na literatura os que identificaram influências de música, de odores, de cores, de iluminação, de configurações do espaço físico e de estímulos relacionados diretamente a situações de compra, como espaço e exposição dos produtos, anúncios e sinalizações (Bailey \& Areni, 2006; Bawa, Landwehr, \& Krishna, 1989; Chevalier, 1975; Cox, 1970; Diehl \& Poynor, 2010; Frank \& Massy, 1970; Herrmann, et al, 2012; Hussain \& Shaheed, 2015; Kotzan \& Evanson, 1969; Lamberton \& Diehl, 2013; Lemon \& Nowlis, 2002; Madzharov, Block, \& Morrin, 2015; Mattila \& Wirtz, 2001; Pizzi \& Scarpi, 2016; Sevilla \& Townsend, 2016; Singh, Katiyar, \& Verma, 2014; Smith \& Burns, 1996; Triantafillidou, Siomkos, \& Papafilippaki, 2017; Wilkinson, Mason, \& Paksoy, 1982).

Considerando os estudos relacionados aos estímulos que estão ligados diretamente a situações de compra, há um que categoriza os elementos atmosféricos em quatro grupos: (1) exterior da loja, (2) 
interior geral, (3) variáveis de layout e design e (4) variáveis de ponto de compra e decoração (Berman \& Evans, 1995). Turley e Milliman (2000) acrescentam um quinto grupo às categorizações previamente feitas, nomeado de "variáveis humanas", tendências individuais como quantidade de itens comprados e comportamentos de evitação da abordagem dos vendedores. Em "variáveis de ponto de compra e decoração" os autores relacionaram atividades promocionais e de merchandising, como exposições no ponto de venda, sinalizações, decorações de paredão e exibidores de preço - todas diretamente ligadas à exibição de produtos dentro da loja.

Esses estímulos são utilizados para atrair os consumidores a fazer compras por impulso (Abratt \& Goodey, 1990; Brandão, Santos \& Freire, 2016; Prieto, 2010; Silva, Diniz, Gonçalves \& Junior, 2018) e potencialmente impactam nas vendas (Wilkinson, Mason, \& Paksoy, 1982). A localização dos produtos na loja e o espaço dado a eles afetam a decisão de compra (Curhan, 1973; Hussain \& Shaheed, 2015; Sevilla \& Townsend, 2016), e também exercem grandes efeitos nos padrões de tráfego dos clientes e na demanda pelos produtos (Çetin, Mersereau, \& Parlaktürk, 2020). No entanto, as respostas às ativações variam inclusive de acordo com as diferenças entre características de categorias e dos produtos, como o giro, preço e sazonalidade (Inman, Winner \& Ferraro, 2009).

Os primeiros estudos que verificaram respostas ao espaço concedido aos produtos na gôndola, por exemplo, concluíram que o espaço nas prateleiras exerce maior influência em itens de impulso e em produtos de alto giro, que são consumidos quase que diariamente pelas pessoas (Curhan, 1973). Já para produtos básicos e marcas de impulso com baixa aceitação pelo consumidor, a receita marginal adicional obtida com o espaço incremental não é considerada significante (Cox, 1970).

Ainda que a reação positiva dos consumidores frente ao espaço dos produtos na gôndola varie de acordo com características do produto e da loja (Curhan, 1973), não apenas o espaço concedido aos produtos, mas também o layout ${ }^{1}$ gera diferentes impactos no consumidor (Lamberton \& Diehl, 2013; Pizzi \& Scarpi, 2016; Simonson \& Winer, 1992). Considerando que há produtos que são comprados mais frequentemente, com intervalos menores entre uma aquisição e outra, a probabilidade de compras não planejadas desta categoria é maior do que para as de menor frequência, caso estejam em exibição (Inman, Winer, \& Ferraro, 2009). Estudo específico com itens de hortifruti, considerados de alta frequência, verificou que o efeito da qualidade da localização da exposição é maior ainda para os itens sazonais (Curhan, 1974), enquanto estudo recente realizado em um supermercado concluiu que o layout da loja e as promoções de vendas influenciam positivamente o comportamento de compra por impulso (Sondakh \& Kalangi, 2020).

Ainda dentro do contexto de exibição de produto, a escolha do produto a ser apresentado em displays também foi objeto de investigação com o intuito de compreender os impactos gerados na demanda por produtos considerando-se dois efeitos. 0 primeiro, é o efeito de aumento de demanda, em que o display potencializa a demanda ao atrair a atenção de consumidores que não comprariam a categoria. 0 segundo é o chamado efeito de substituição, em que o display pode induzir clientes a substituir o produto que originalmente eles preferiam. Assim, ao escolher um produto de uma categoria específica, as possibilidades para otimizar os efeitos de exposição em um display promocional se baseiam em um conjunto eficiente desenhado em termos de popularidade e margem dos produtos, e que a melhor escolha em relação ao posicionamento do display depende, em certa medida, da atratividade do corredor e de muitos parâmetros técnicos relacionados às categorias dos produtos (Çetin et al., 2020).

0 efeito de resposta quando exibições são combinadas com informações de preço e respostas a anúncios (Lemon \& Nowlis, 2002; Sondakh \& Kalangi, 2020; Wilkinson, Mason, \& Paksoy, 1982) também foram investigados, e elas podem variar para diferentes tipos de itens (Curhan, 1974) e perfis de marcas (Lemon \& Nowlis, 2002). Os preços dos produtos ativados em corredores principais, como o central e o de entrada, por exemplo, podem ser percebidos diferentemente a partir das políticas de merchandising executadas (Smith \& Burns, 1996).

Assim, o efeito de resposta dos estímulos das exibições e espaços em loja pode ser diferente de acordo com as variedades nas características dos produtos (Chevalier, 1975; Curhan, 1974; Stoll, Baecke \& Kenning 2008), perfis de marcas (Lemon \& Nowlis, 2002) e localização na loja. 0 volume e o sortimento ativado podem influenciar diretamente nas percepções do consumidor (Diehl \& Poynor, 2010; Smith \& Burns, 1996). 


\section{MÉTODO}

0 presente estudo tem natureza descritiva - por descrever tendências de um grupo e pretender mostrar as dimensões do fenômeno em um contexto específico - e correlacional - por quantificar graus de associação e relações entre variáveis. 0 método adotado foi de abordagem quantitativa, e envolveu a aplicação de questionário online, por um período delimitado, tendo ficado disponível na semana anterior e posterior ao Natal, durante 10 dias.

\section{Participantes}

Participaram deste estudo 131 consumidores, na cidade do Rio de Janeiro, com média de idade de 44,2 anos $(D P=10,88)$, sendo $51,1 \%$ mulheres e $48,9 \%$ homens. A amostra contém pessoas de todas as escolaridades: $1,5 \%$ com ensino fundamental; $6,1 \%$ com ensino médio completo; $4,6 \%$ com ensino superior incompleto; $28,2 \%$ com ensino superior completo; $13 \%$ com pós-graduação incompleta e $46,6 \%$ das pessoas possuía pós-graduação completa.

\section{Instrumentos}

Foi aplicado um questionário online, disponibilizado em endereço de uma plataforma virtual na internet. Ao clicar no link, os participantes eram direcionados para o questionário que iniciava com questões sobre atividades de compra recentes, tais como: se esteve em mercados nas semanas préNatal e pré-Réveillon, se efetuou compra, quantidade de itens comprados. A seguir, participantes responderam se costumavam ir sozinhos ou acompanhados ao mercado, e se costumavam fazer lista de compras. Questões sobre lembrança de ativações promocionais e sobre influência de tais ativações também foram feitas. 0 primeiro bloco de perguntas terminava com questões sociodemográficas (sexo, idade em anos, escolaridade, estado civil).

Por último, o questionário continha uma escala para aferir a tendência que as pessoas têm de comprar por impulso. Para medir esse traço, utilizou-se uma versão brasileira do instrumento de Rook e Fisher (1995), a Escala de Compra por Impulso (Aquino, Natividade \& Lins, 2020). 0 instrumento contém nove itens avaliados em uma escala Likert de sete pontos. Quanto maiores os escores, maior a tendência a comprar por impulso. Na presente amostra, a consistência interna da escala apresentou alfa de Cronbach de 0,80 .

\section{Procedimentos de coleta}

Participantes foram recrutados atendendo a convites por e-mail e redes sociais; e responderam ao questionário disponibilizado na internet via plataforma online. A amostra foi não-probabilística por conveniência, não tendo sido realizado qualquer cálculo amostral para definir um número mínimo. No entanto, para uma conduta adequada de análises estatísticas, buscou-se a maior quantidade de respondentes possível durante o período de dez dias compreendidos antes e depois de 25 de dezembro.

\section{Procedimentos éticos}

$\mathrm{Na}$ primeira página do site em que o questionário estava disponível foi apresentado um resumo da proposta, garantindo o anonimato dos participantes, a confidencialidade das respostas e a livre deliberação em participar da pesquisa.

\section{Procedimentos de análise}

Utilizando o software SPSS, foram realizadas estatísticas descritivas, análises de correlação de Pearson e testes $t$ de Student. Além disso, associações entre as variáveis foram testadas usando o quiquadrado. Do total de respondentes, sete foram excluídos por declararem não terem ido ao mercado no período solicitado. 0 questionário foi configurado para não permitir respostas omissas aos itens da escala, desse modo, não foi necessário realizar procedimentos de transformação de casos omissos. 


\section{RESULTADOS}

Inicialmente, foram testadas as relações entre tendências individuais e dados sociodemográficos. Por meio de coeficientes de correlação $r$ de Pearson, compra por impulso e idade não se correlacionaram de forma significativa estatisticamente. Tendo em vista que a literatura sugere diferenças entre grupos na compra por impulso (Dittmar, 2005; Grewal et al., 2003; Lins \& Pereira, 2011; Tifferet \& Hernstein, 2012), novas análises também foram realizadas separando homens e mulheres, e os resultados permaneceram não significativos. Considerando os dados comportamentais coletados, a amostra foi dividida entre o grupo dos que não tinham planejado comprar e o grupo dos que tinham planejado comprar; e, neste caso, foram encontradas correlações significativas entre compra por impulso e idade. A correlação foi positiva no grupo que declarou não ter planejado comprar panetone, mas comprou $(r=0,58 ; p<0,05)$; e foi negativa no grupo que declarou ter planejado e comprado $(r=-0,42, p<0,05)$.

Adicionalmente, por meio de testes $t$ de Student, verificou-se se houve significativas diferenças de média de compra por impulso entre sexo feminino e masculino, e entre os grupos das pessoas que declararam ter hábito de fazer lista de compras e pessoas que declararam não ter esse hábito. Também foi realizada uma ANOVA a fim de verificar diferenças de médias entre faixas etárias. Na presente amostra, essas diferenças de média não foram estatísticamente significativas.

Em seguida, foi aplicado um teste não paramétrico (qui-quadrado) para verificar a distribuição dos sujeitos por diversas condições, e, dessa maneira, avaliar a associação existente entre a ocorrência de planejamento de compra e variáveis relacionadas à ativação promocional no ponto de vendas. As pessoas foram perguntadas se tinham planejado comprar panetone e/ou chocotone antes da sua ida ao mercado, e marcaram se tinham ido ao mercado já com a intenção prévia de comprar este(s) produto(s). Como visto nas tabelas 1, 2 e 3, algumas associações foram significativas.

Tabela 1

Associações entre planejamento da compra e lembrança de ativação promocional

\begin{tabular}{|c|c|c|c|c|c|}
\hline & \multicolumn{5}{|c|}{$\begin{array}{l}\text { Lembra de ter visto/ouvido no supermercado algum tipo de oferta, } \\
\text { promoção, anúncio sobre panetone e/ou chocotone num espaço especial da } \\
\text { loja? }\end{array}$} \\
\hline & $\begin{array}{l}\text { num espaço } \\
\text { especial da } \\
\text { loja (por ex.: } \\
\text { ilha de } \\
\text { produtos) }\end{array}$ & $\begin{array}{c}\text { na } \\
\text { prateleira } \\
\text { convencio- } \\
\text { nal }\end{array}$ & $\begin{array}{c}\text { num grande } \\
\text { cartaz } \\
\text { destacando } \\
\text { preço ou } \\
\text { produto }\end{array}$ & $\begin{array}{l}\text { em anúncio no } \\
\text { encarte ou } \\
\text { folheto } \\
\text { disponível na } \\
\text { entrada }\end{array}$ & $\begin{array}{l}\text { através de } \\
\text { locutor } \\
\text { anunciando } \\
\text { preço ou } \\
\text { produto }\end{array}$ \\
\hline $\begin{array}{l}\text { Não, não tinha } \\
\text { planejado e não } \\
\text { comprei }\end{array}$ & 39 & 15 & 6 & 8 & 6 \\
\hline $\begin{array}{l}\text { Não, não tinha } \\
\text { planejado, mas } \\
\text { comprei }\end{array}$ & 5 & 5 & 3 & 3 & 3 \\
\hline $\begin{array}{l}\text { Sim, tinha planejado } \\
\text { e comprei }\end{array}$ & 15 & 8 & 5 & 2 & 0 \\
\hline $\begin{array}{l}\text { Sim, tinha planejado, } \\
\text { mas não comprei }\end{array}$ & 13 & 3 & 3 & 0 & 4 \\
\hline$\chi^{2}(\mathrm{gl})$ & $12,32(4)$ & $6,34(4)$ & $9,56(4)$ & $3,07(4)$ & $19,40(4)$ \\
\hline$p$ value & 0,015 & 0,175 & 0,049 & 0,546 & 0,001 \\
\hline
\end{tabular}


Tabela 2

Associações entre planejamento de compra e percepção de influência da ativação

\begin{tabular}{|c|c|c|c|c|c|c|}
\hline & \multicolumn{6}{|c|}{$\begin{array}{c}\text { Das ativações promocionais que o supermercado tinha de } \\
\text { panetone/chocotone, } \\
\text { o que influenciou... }\end{array}$} \\
\hline & $\begin{array}{c}\text { nada } \\
\text { influenciou }\end{array}$ & $\begin{array}{l}\text { espaço } \\
\text { especial } \\
\text { da loja }\end{array}$ & $\begin{array}{l}\text { prateleira } \\
\text { convencio } \\
\text { nal }\end{array}$ & $\begin{array}{c}\text { locutor } \\
\text { anuncian } \\
\text { do preço } \\
\text { ou } \\
\text { produto }\end{array}$ & $\begin{array}{l}\text { anúncio em } \\
\text { encarte ou } \\
\text { folheto } \\
\text { disponível na } \\
\text { entrada }\end{array}$ & $\begin{array}{c}\text { grande } \\
\text { cartaz } \\
\text { destacando } \\
\text { preço ou } \\
\text { produto }\end{array}$ \\
\hline $\begin{array}{l}\text { Não, não tinha } \\
\text { planejado e não } \\
\text { comprei }\end{array}$ & 0 & 0 & 0 & 0 & 0 & 0 \\
\hline $\begin{array}{l}\text { Não, não tinha } \\
\text { planejado, mas } \\
\text { comprei }\end{array}$ & 4 & 5 & 3 & 2 & 1 & 2 \\
\hline $\begin{array}{l}\text { Sim, tinha planejado } \\
\text { e comprei }\end{array}$ & 10 & 8 & 4 & 0 & 1 & 3 \\
\hline $\begin{array}{l}\text { Sim, tinha planejado, } \\
\text { mas não comprei }\end{array}$ & 0 & 0 & 0 & 0 & 0 & 0 \\
\hline$\chi^{2}(\mathrm{gl})$ & $42,42(4)$ & $35,60(4)$ & $18,11(4)$ & $14,60(4)$ & & $29,10(3)$ \\
\hline$p$ value & $<0,001$ & $<0,001$ & 0,001 & 0,006 & & 0,000 \\
\hline
\end{tabular}

Tabela 3

Associações entre compras efetivadas e percepção da influência da ativação promocional

\begin{tabular}{lcccccc}
\hline \multicolumn{7}{c}{ Das ativações promocionais que o supermercado tinha de } \\
panetone/chocotone, \\
o que influenciou...
\end{tabular}

Por último, considerando apenas o grupo de pessoas que declarou ter planejado comprar panetone/chocotone, a amostra foi subdividida entre quem comprou algum produto e quem não comprou. Dos 38 indivíduos que compraram, 10\% declararam ter comprado mais do que o planejado $(\chi 2=60,53 ; p<0,001)$. Também entre os respondentes que disseram ter comprado panetone ou chocotone, as respostas sobre finalidade da compra ficaram assim divididas: $52,6 \%$ afirmaram que a compra foi para consumo próprio, 18,4\% para presentear, e 28,9\% marcaram ambas as opções. 


\section{DISCUSSÃO}

O objetivo deste estudo foi verificar se ativações promocionais de panetones e chocotones durante o final do ano estimulam o consumidor a comprar itens sem planejamento. Partiu-se de um desenho descritivo para realizar associações de lembranças, percepção e influência da comunicação em supermercados, a fim de avaliar se as ativações promocionais no ambiente de compra influenciam as decisões do consumidor. Este estudo tem o diferencial de diversidade amostral formada não só de universitários, como é comum em estudos com variáveis psicológicas. Essa variedade amostral nos permite compreender melhor os achados, sob essa perspectiva.

Tendo em vista que a literatura sugere que a tendência a compra por impulso é mais alta em pessoas do sexo feminino (Aquino, Natividade, \& Lins, 2019; Dittmar, 2005; Grewal et al., 2003; Tifferet \& Hernstein, 2012), e diminui com o aumento da idade (Dittmar, 2005), o resultado encontrado não corrobora esses achados. Ainda que a caracterização da amostra possa ter enviesado as respostas, vale ressaltar que a particularidade dessa investigação suscitou novos resultados. Além disso, a estratégia adotada de analisar os dados separando o grupo entre pessoas que não tinham planejado comprar e pessoas que tinham planejado apontou para correlações significativas entre compra por impulso e idade.

A tendência de comprar impulsivamente aumentou à medida que a idade crescia no grupo que não tinha planejado a compra, porém comprou. Essa forte correlação pode indicar que a decisão de compra tende a ser mais relacionada a tendências individuais e características pessoais que interferiram na decisão. Supõe-se que pessoas mais velhas que compraram panetone ou chocotone mesmo sem ter planejado podem ter tido outras motivações para essa decisão, e esse comportamento está correlacionado com a tendência individual de compra por impulso, fazendo essas pessoas agirem de forma espontânea e quase irrefletida, estimuladas pela proximidade física com o objeto.

Por outro lado, entre pessoas que declaram ter planejado e comprado, a correlação da tendência a compra por impulso com idade foi negativa, indicando que, nesse grupo, à medida que a idade avançava, a tendência a comprar impulsivamente diminuía. Isso corrobora a literatura e indica que o planejamento a respeito da compra pode ser mais decisivo sobre a decisão final de compra dos consumidores mais velhos (Aquino, Natividade \& Lins, 2019; Dittmar, 2005).

Ao verificar que a diferença de média de compra por impulso entre sexo feminino e masculino, e entre os grupos das pessoas que declararam ter hábito de fazer lista de compras e pessoas que declararam não ter esse hábito não foram significativas, este estudo não corrobora o que diz a literatura recente (Aquino, Natividade \& Lins, 2019). Desta forma, levantam-se questões não apenas sobre tamanho da amostra, como também sobre seu enviesamento. Considerando que somente 1,5\% dos participantes tem menos de 24 anos, é compreensível que haja mais conscienciosidade do que impulsividade na tendência de compra por impulso. 0 alto grau de escolaridade (59\% dos respondentes tinha pós-graduação) também pode ser um fator tendencioso para as respostas desejáveis socialmente, pois é razoável supor que esse público tende a refletir mais sobre suas declarações em questionários de autorresposta.

Ao avaliar associações existentes entre algumas variáveis, as que foram significativas indicam que o planejamento prévio pode ser mais relevante para esses tipos de amostra e de produto do que qualquer ativação ou motivação espontânea. A maioria das pessoas que compraram panetone e/ou chocotone, compraram exatamente o que tinham planejado. 0 número de indivíduos que declarou não ter planejado e não comprou, mesmo tendo se lembrado e visto as ativações promocionais, foi maior do que o número de pessoas que afirmou não ter planejado e ter comprado. Isso aponta para o quanto o planejamento foi mais forte do que a exposição às ativações.

Aprofundando estes achados e as significâncias estatísticas, identificamos algumas tendências comportamentais. A primeira, nos indica que quem declarou não ter planejado comprar panetones ou chocotones, mas comprou, lembrou de ter visto ativações promocionais com espaços especiais nas lojas (por ex.: ilha de produtos), grandes cartazes anunciando preços ou produtos, ou lembrou de ter ouvido locução de funcionário anunciando preços ou produtos. Supõe-se que tais ativações são mais associadas a uma compra não planejada, apontando para estes estímulos como mais provavelmente lembrados pelo consumidor, pois a lembrança de ter visto alguma ativação promocional foi bem mais 
alta nos indivíduos que tinham planejado comprar do que naqueles que não tinham planejado.

A declaração de ter sido influenciado por alguma comunicação também foi mais incidente entre os que declaram terem ido ao mercado já com intenção de comprar o produto. Isso pode confirmar que, ao planejar, o consumidor tende a observar mais as ações de comunicação do produto no ponto de venda do que o consumidor que não planejou. A exposição pode ser mais apelativa entre os potenciais compradores do que entre os que não tem intenção de comprar. As ativações promocionais citadas como influenciadoras por pessoas que declararam ter comprado panetones ou chocotones mesmo sem ter planejado, foram o espaço especial da loja, a prateleira convencional, o locutor e o grande cartaz anunciando preço ou produto. Adicionalmente, quem declarou que comprou além do que havia planejado, indicou ser influenciado pela arrumação da prateleira convencional, corroborando estudo sobre o quanto a atmosfera do ambiente impacta na quantidade de dinheiro que as pessoas gastam e no número de itens que compram (Turley \& Milliman, 2000). Ou seja, o comportamento de não planejar parece deixar os consumidores mais vulneráveis a estímulos provocados por ativações promocionais no ponto de venda. Já as pessoas que, de alguma forma, planejaram comprar, aparentemente foram menos suscetíveis a essas influências.

As análises sugerem uma possível limitação na forma de formular a pergunta: o uso da palavra "influência" na pergunta sobre a ativação pode ter gerado um viés uma reatância psicológica (Brehm, 1989). A teoria da reatância (Brehm, 1989) aponta que pessoas motivadas pela percepção de cerceamento de uma liberdade comportamental, tendem a procurar restaurar tal liberdade que sentem como ameaçada. Seria como se, ao ler o enunciado, pensassem que não são influenciadas por coisa alguma, e assim, resistissem a admitir que o foram. 0 enunciado era "Das ativações promocionais que o supermercado tinha de panetone/chocotone, o que influenciou...". Se a forma escrita fosse "o que te estimulou", talvez amenizasse o desconforto que a expressão "ser influenciado" pode causar. 0 uso de outro vocábulo poderia ter mensurado com maior eficácia e menor viés o que se pretendia.

Um dado a ser considerado é que $47,3 \%$ da amostra comprou panetone ou chocotone para presentear. Ao supor que esses são itens eventualmente comprados para dar para outras pessoas, podemos atribuir o planejamento da compra de itens sazonais a um exercício do papel social do consumidor. Isso suscita a conclusão de que a aquisição é motivada especialmente pelo caráter de meta atingida, ou tarefa a ser completada, e refletindo uma satisfação de cumprir papeis quando vai às compras (Aquino \& Natividade, 2020; Arnold \& Reynolds, 2003; Cinjarevic et al, 2011).

Outra limitação deste estudo foi o tamanho amostral. Optou-se por adotar uma temporalidade transversal de curto período, a fim de fazer um recorte do objeto estudado dentro de um tempo determinado, que fosse mais apropriado metodologicamente para atingir o objetivo proposto. Então, o curto espaço de tempo e a natural sobrecarga do período natalino, impediram de alcançar mais respondentes. Amostras numericamente pequenas trazem consigo limitações estatísticas, porém podem levantar dados que sejam explorados de forma mais abrangente futuramente, possibilitando desenvolver e ajustar conceitos, considerando outros aspectos relacionados ao foco do estudo.

Por fim, o aumento do número de frequentadores em supermercados na época da coleta pode ter influenciado a maneira como os consumidores responderam aos apelos de comunicação. Algumas emoções negativas relacionadas à aglomeração de pessoas - que faz aumentar número de filas e piora a circulação nas lojas - podem influenciar negativamente tanto o impacto das ativações promocionais em locais de alta densidade humana, quanto a reatividade dos consumidores a circunstâncias externas (Aquino \& Lins, 2020; Ferreira, Brandao \& Bizarrias, 2015).

\section{CONSIDERAÇÕES FINAIS}

0 produto em si pode ter determinado a maioria das respostas, embora tenha sido elemento fundamental do objetivo proposto. Se nem todas as pessoas gostam, consomem ou presenteiam com panetone e chocotone na época de fim de ano, o questionário poderia ter sido pensado de forma a identificar esse viés. Futuros estudos devem abordar a preferência de produtos natalinos antes de entender as ativações que influenciam a decisão de compra no ponto de venda. A utilização de vídeo etnográfico para pesquisas pode ser uma ferramenta útil para avaliação in loco do comportamento e tomada de decisão em datas sazonais em locais com ativação promocional. 
A ausência de literatura abrangente sobre ativações de produtos sazonais em supermercados no Brasil aponta para a necessidade de novos estudos aprofundando outras questões. Por exemplo, futuros estudos podem enfocar se a aglomeração de consumidores traz consequências no ambiente de loja, uma vez que a densidade humana influencia a percepção de fatores como layout e ambiente (Eroglu, Machleit \& Barr, 2005).

Com relação a implicações gerenciais, tomando como base os resultados deste estudo, existe oportunidade para que tanto executivos de varejo quanto fabricantes de panetones e/ou chocotones revisem suas estratégias promocionais. Se a incidência de compra planejada é mais comum do que a compra não planejada, os espaços promocionais das lojas onde predominam as ativações de panetone podem ser otimizados, dando espaço para que outros produtos mais propícios à compra por impulso tenham destaque. Por outro lado, pode-se desenvolver uma comunicação reforçando mais a associação do panetone e/ou chocotone com a ideia de presentear, que parece ser uma das principais motivações de compra por impulso no período natalino.

Estudos como este, que consideram variáveis comportamentais e psicológicas, reforçam que pessoas diferentes têm experiências diferentes (Lakoff \& Johnson, 2003), e tais experiências impactam suas decisões de compra. A compra impulsiva dos consumidores depende não somente do contexto dentro do qual eles vivem - considerando renda, classe social, região etc. -, como também de tendências individuais e hábitos de consumo. $\mathrm{O}$ valor atribuído ao produto e o custo monetário também devem ser levados em conta em novas abordagens do tema, pois preços e promoções tendem a ser elementos que fazem pessoas comprarem por impulso em supermercados (Flores, 2018).

Para compreender completamente os padrões da compra impulsiva de produtos específicos durante um período determinado, é necessário também considerar, além das ativações promocionais em lojas físicas, outras variáveis. Conexões e interações sociais, e até mesmo o valor atribuído aos significados do período festivo, podem ser fatores determinantes dos comportamentos de compra por impulso de panetone ou chocotones. Ainda que encontrar panetone nos supermercados remeta à atmosfera típica do período natalino (Chianezi \& Oliveira, 2020), os níveis de importância atribuídos a esse fenômeno devem ser considerados. Isto insere, inclusive, a necessidade de testar esse objetivo considerando compras impulsivas pela internet, que já se apresenta como um novo ambiente de compra, com ativações e público específicos.

"Então é Natal... e o que você fez?" Tradicionalmente, os brasileiros compram panetones e chocotones para presentear ou compor a própria mesa durante as festas de fim de ano (Chianezi \& Oliveira, 2020). Porém, ainda que consumidores incluam ou não essas compras sazonais em seus planejamentos, comprar espontaneamente, motivados pela proximidade física do objeto e atendendo a estímulos momentâneos envolve outras variáveis externas, que não apenas o impacto da ativação promocional. Aparentemente, há um conjunto de variáveis psicológicas, sociais, estratégicas e promocionais que devem ser consideradas para entender a suscetibilidade a influências no ponto de venda ativado. A compra por impulso é um vasto campo para estudos que enfatizem maior diversidade de variáveis, possibilitando a ampla integração de cientistas comportamentais dos campos de Administração, de Marketing e de Psicologia.

\section{Referências}

Abratt, R., \& Goodey, S. D. (1990). Unplanned Buying and In-store Stimuli in Supermarkets. Managerial and Decision Economics, 11, 111-121. Retirado de https://www.jstor.org/stable/2487460

Aquino, S. D. \& Lins, S. (2020). Compras Excessivas como Estratégia de Enfrentamento (CEEE) propriedades psicométricas iniciais de um novo instrumento. Painel apresentado na $50^{a}$ Reunião Anual da Sociedade Brasileira de Psicologia, Brasília, DF. doi: 10.13140/RG.2.2.26550.52803 Retirado https://www.researchgate.net/publication/345814908_Compras_Excessivas_como_Estrategia_ de_Enfrentamento_CEEE - propriedades_psicometricas_iniciais_de_um_novo_instrumento 
Aquino, S. D., \& Natividade, J. C. (2020a). Motivações hedônicas para compras: Adaptação de um instrumento para o contexto brasileiro. Pôster apresentado na 50a Reunião Anual da Sociedade Brasileira de Psicologia, Brasília, DF. doi: 10.13140/RG.2.2.15122.68803. Recuperado de https://www.researchgate.net/publication/345328785_Motivacoes_Hedonicas_para_Compras__Adaptacao_de_um_Instrumento_para_o_contexto_brasileiro

Aquino, S. D., \& Natividade, J. C. (2020b). Relações entre os Cinco Grandes Fatores da personalidade e motivações hedônicas para compras. Apresentação oral em Sessão coordenada apresentada na 50 a Reunião Anual da Sociedade Brasileira de Psicologia, Brasília, DF. Congresso Online. http://www.sbponline.org.br/anais-e-resumos

Aquino, S. D., Natividade, J. C., \& Lins, S. (2019). Sua personalidade vai ao shopping? Relações entre personalidade e a compra por impulso. Psico, 50(4), 31905. doi: 10.15448/19808623.2019.4.31905

Aquino, S. D., Natividade, J. C., \& Lins, S. L. B. (2020). Validity evidences of the Buying Impulsiveness Scale in the Brazilian Context. Psico-USF, 25(1), 15-25. doi: 10.1590/1413-82712020250102

Arnold, M. J., \& Reynolds, K. E. (2003). Hedonic shopping motivations. Journal of retailing, 79(2), 77-95. doi: 10.1016/S0022-4359(03)00007-1

Babin, B. J., Darden, W. R., \& Griffin, M. (1994). Work and/or fun: Measuring hedonic and utilitarian shopping value. Journal of Consumer Research, 20(4), 644-656. Recuperado de https://www.jstor.org/stable/2489765

Bailey, N., \& Areni, C. S. (2006). When a few minutes sound like a lifetime: Does atmospheric music expand or contract perceived time? Journal of Retailing, 82(3), 189-202. doi: 10.1016/j.jretai.2006.05.003

Banat, A., \& Wandebori, H. S. T. (2012). Store Design and Store Atmosphere Effect on Customer Sales per Visit Economics. Management and Behavioral Science, 84-89. Recuperado de http://psrcentre.org/images/extraimages/10\%201012545.pdf

Batra, R., \& Ahtola, O. T. (1991). Measuring the hedonic and utilitarian sources of consumer attitudes. Marketing Letters, 2(2), 159-170. doi: 10.1007/BF00436035

Bawa, K., Landwehr, J. T., \& Krishna, A. (1989). Consumer response to retailers' marketing environments: An analysis of coffee purchase data. Journal of Retailing, 65(4), 471-495.

Beatty, S. E., \& Ferrell, M. E. (1998). Impulse buying: Modeling its precursors. Journal of retailing, 74(2), 169-191. doi: 10.1016/S0022-4359(99)80092-X

Berman, B., \& Evans, J. R. (1995). Retail Management: A strategic approach. (N. Prentice-Hall, Inc., Englewood Cliffs, Ed.) (6th ed.).

Bhardwaj, V., \& Manchiraju, S. (2017). The role of impulse buying, hedonism, and consumer knowledge towards sustainable consumption of fast fashion. International Textile and Apparel Association (ITAA) Annual Conference Proceedings. St. Petersburg, Florida. Recuperado de https://lib.dr.iastate.edu/itaa_proceedings/2017/presentations/151/

Bloch, P. H., Sherrell, D. L., \& Ridgway, N. M. (1986). Consumer search: An extended framework. Journal of Consumer Research, 13(1), 119-126. doi: 10.1086/209052

Brandão, M. M., Santos, E. B. A., \& Freire, O. (2016). A Relação entre controle situacional, crowding e características do ambiente de loja: a influência na satisfação e compra por impulso. In CLAV 2016.

Brehm, J. W. (1989). Psychological Reactance: Theory and Applications: Advances in Consumer Research. In Srull, T. K (Ed.), Association for Consumer Research (Vol. 16, pp- 72-75). Provo, UT.

Çetin, O., Mersereau, A. J., \& Parlaktürk, A. K. (2020). Management and effects of in-store promotional displays. Manufacturing and Service Operations Management, 22(3), 481-494. doi: $10.1287 /$ msom.2018.0749

Chang, H. H., \& Fang, W. (2012). The effects of shopping value on retail outcomes: A comparison between department stores and hypermarkets. The Service Industries Journal, 32(14), 22492263. doi: 10.1080/02642069.2011.582494

Chevalier, M. (1975). Increase in sales due to in-store display. Journal of Marketing Research, 12(4), 426-431. doi: 10.1177/002224377501200406 
Chianezi, M. \& Oliveira, R. (2020, Agosto 28). Então é Natal? Pandemia acelera produção e panetones já aparecem nas prateleiras. Jornal Mídiamax. Recuperado de https://www.midiamax.com.br/cotidiano/economia/2020/pandemia-acelera-producao-epanetones-ja-aparecem-nas-prateleiras-em-pleno-agosto

Činjarević, M., Tatić, K., \& Petrić, S. (2011). See it, like it, buy it! Hedonic shopping motivations and impulse buying. Economic Review: Journal of Economics \& Business/Ekonomska Revija: Casopis za Ekonomiju i Biznis, 9. Recuperado de: https://www.econstor.eu/handle/10419/193794

Cox, K. (1970). The effect of shelf space upon sales of branded products. Journal of Marketing Research, 7, 55-58. doi: 10.1177/002224377000700106

Curhan, R. C. (1973). Shelf space allocation and profit maximization in mass retailing. Journal of Marketing, 37, 54-60. doi: 10.1177/002224297303700308

Curhan, R. C. (1974). The effects of merchandising and temporary promotional activities on the sales of fresh fruits and vegetables in supermarkets. Journal of Marketing Research, 11(August), 286294. doi: $10.1177 / 002224377401100307$

Diehl, K., \& Poynor, C. (2010). Great expectations?! Assortment size, expectations, and satisfaction. Journal of Marketing Research, 47(2), 312-322. doi: 10.1509/jmkr.47.2.312

Dittmar, H. (2005). Compulsive buying - a growing concern? An examination of gender, age, and endorsement of materialistic values as predictors. British Journal of Psychology, 96(4), 467-491. doi: 10.1348/000712605X53533

Eroglu, S. A., Machleit, K., \& Barr, T. F. (2005). Percepção do apinhamento no varejo e satisfação com compras: 0 papel dos valores de compras. Journal of Business Research, 58, 1146-1153. doi:

Ferreira, M. C. O., Brandão, M. M., \& Bizarrias, F. S. (2017). Understanding consumer's responses to negative emotions related to crowding on satisfaction and impulse purchase in retail: the mediating role of coping. Revista de Administração (São Paulo), 52(4), 431-442. doi: 10.1016/j.rausp.2017.08.005

Frank, R. E., \& Massy, W. F. (1970). Shelf position and space effects on sales. Journal of Marketing Research, 7(1), 59-66. doi: 10.1177/002224377000700107

Grewal, D., Baker, J., Levy, M., \& Voss, G. B. (2003). The effects of wait expectations and store atmosphere evaluations on patronage intentions in service-intensive retail stores. Journal of Retailing, 79, 259-268. doi: 10.1016/j.jretai.2003.09.006

Herrmann, A., Zidansek, M., Sprott, D. E., \& Spangenberg, E. R. (2012). The power of simplicity: Processing fluency and the effects of olfactory cues on retail sales. Journal of Retailing, 89(1), 3043. doi: $10.1016 /$ j.jretai.2012.08.002

Hirschman, E. C., \& Holbrook, M. B. (1982). Hedonic consumption: Emerging concepts, methods and propositions. The Journal of Marketing, 92-101. doi: 10.1177/002224298204600314

Hussain, R., \& Ali, M. (2015). Effect of store atmosphere on consumer purchase intention. International Journal of Marketing Studies, 7(2), 35-43. doi: 10.2139/ssrn.2588411

Inman, J. J., Winer, R. S., \& Ferraro, R. (2009). The interplay among category characteristics, customer characteristics, and customer activities on in-store decision making. Journal of Marketing, 73, 19-29. doi: $10.1509 /$ jmkg.73.5.19

Lemon, K. N., \& Nowlis, S. M. (2002). Developing synergies between promotions and brands in different price-quality tiers.Journal of Marketing Research,39(2), 171-185. doi: 10.1509/jmkr.39.2.171.19089

Khachatryan, H., Rihn, A., Behe, B., Hall, C., Campbell, B., Dennis, J., \& Yue, C. (2018). Visual attention, buying impulsiveness, and consumer behavior. Marketing Letters, 29(1), 23-35. doi: $10.1007 / \mathrm{s} 11002-018-9446-9$

Kotler, P. (1973). Atmospheric as a Marketing Tool. Journal of Retailing, 49(4), 48-64. Recuperado de: http://belzludovic.free.fr/nolwenn/Kotler\%20\%20Atmospherics\%20as\%20a\%20marketing\%20tool\%20\%20(cit\%C3\%A9\%20171)\%20\%201973.pdf

Kotzan, J. A., \& Evanson, R. V. (1969). Responsiveness of drug store sales to shelf space allocations. Journal of Marketing Research, 6(4), 465-469. doi: 10.1177/002224376900600413 
Lamberton, C. P., \& Diehl, K. (2013). Retail choice architecture: The effects of benefit- and attributebased assortment organization on consumer perceptions and choice. Journal of Consumer Research, 40, 393-411. doi: 10.1086/671103

Laroche, M., Saad, G., Kim, C., \& Browne, E. (2000). A cross-cultural study of in-store information search strategies for a christmas gift. Journal of Business Research, 49(2), 113-126. doi: 10.1016/S0148-2963(99)00008-9

Lins, S. L. B., \& Pereira, R. de C. de F. (2011). Comprou sem querer? Entenda o porquê! Revista Brasileira de Gestão de Negócios, 13(41), 376-395. doi: 10.13140/RG.2.2.12531.12326

Madzharov, A. V., Block, L. G., \& Morrin, M. (2015). The cool scent of power: Effects of ambient scent on consumer preferences and choice behavior.Journal of Marketing, 79(1), 83-96. doi: $10.1509 /$ jm.13.0263

Flores, J. A. A. Factores que motivan las compras impulsivas y el gasto con las tarjetas de crédito en los hipermercados, Trujillo-2018. Tese de Doutorado, Universidad César Vallejo. Facultad de Ciencias Empresariales, Trujillo, Peru. Recuperado em dezembro de 2020 de https://hdl.handle.net/20.500.12692/24328

Mattila, A. S., \& Wirtz, J. (2001). Congruency of scent and music as a driver of in-store evaluations and behavior. Journal of Retailing, 77(2), 273-289. doi: 10.1016/S0022-4359(01)00042-2

Nielsen Holdings N.V. (NYSE: NLSN). The Nielsen Company. (2017). Execução consciente: O gatilho certo para atrair os shoppers. Recuperado de https://www.nielsen.com/br/pt/insights/article/2017/execucao-consciente-o-gatilho-certopara-atrair-os-shoppers/

Nielsen Holdings N.V. (NYSE: NLSN). The Nielsen Company. Nielsen Media Research (2015). Nielsen series: A hora certa de ativar o shopper. Recuperado de: https://www.nielsen.com/wpcontent/uploads/sites/3/2019/04/NielsenSeries-Shopper-2015.pdf

Pizzi, G., \& Scarpi, D. (2016). The effect of shelf layout on satisfaction and perceived assortment size: An empirical assessment. Journal of Retailing and Consumer Services, 28, 67-77. doi: 10.1016/j.jretconser.2015.08.012

Point of Purchase Advertising International. POPAI. (2012). Shopper engagement study - Media topline report. Recuperado de http://www.popai.cz/files/publishing/shopper-engagementstudy_5165.pdf

Prieto, J. (2010). Merchandising: La seducción en el punto de venta. 2a ed. México: Ecoe Ediciones.

Rook, D. W., \& Fisher, R. J. (1995). Normative influences on impulsive buying behavior. Journal of Consumer Research, 22(3), 305. doi: 10.1086/209452

Sevilla, J., \& Townsend, C. (2016). The Space-to-Product Ratio Effect: How Interstitial Space In fluences Product Aesthetic Appeal, Store Perceptions, and Product Preference. Journal of Marketing Research, 53(5), 665-681. doi: 10.1509/jmr.13.0601

Silva, W. T., Diniz, L. M., Silva, W. R., Gonçalves Filho, C., \& Junior, C. L. N. (2018). Os principais fatores que impulsionam a compra por impulso. Working paper apresentado no Congresso LatinoAmericano de Varejo (CLAV 2018). Retirado de http://bibliotecadigital.fgv.br/ocs/index.php/clav/clav2018/paper/view/6803/1923

Silveira, D (2019, Outubro 13). Faltando mais de dois meses para o Natal, prateleiras de mercados já oferecem $\quad$ panetones. $\quad$ Recuperado de https://g1.globo.com/economia/noticia/2019/10/13/faltando-mais-de-dois-meses-para-onatal-prateleiras-de-mercados-ja-oferecem-panetones.ghtml

Simonson, I., \& Winer, R. S. (1992). The influence of purchase quantity and display format on consumer preference for variety.Journal of Consumer Research,19(1), 133-138. doi: $10.1086 / 209292$

Singh, P., Katiyar, N., \& Verma, G. (2014). Retail shoppability: the impact of store atmospherics \& store layout on consumer buying patterns. International Journal of Scientific \& Technology Research Volume, 3(8), 15-23.

Smith, P. C., \& Curnow, R. (1966). "Arousal Hypothesis" and the effects of music on purchasing behavior. Journal of Applied Psychology, 50(3), 255-256. doi: 10.1037/h0023326 
Smith, P., \& Burns, D. J. (1996). Atmospherics and retail environments: the case of the "power aisle". International Journal of Retail \& Distribution Management, 24(1), 7-14. doi: 10.1108/09590559610107076

Sondakh, P. L. Y., \& Kalangi, J. B. (2020). The influence of sales promotion and store layout on consumer impulsive buying behavior. Case study: Cool supermarket tomohon. Jurnal EMBA: Jurnal Riset Ekonomi, Manajemen, Bisnis dan Akuntans, 8(4), 668-674. doi: 10.35794/emba.v8i4.31003

Soysal, G. P., \& Krishnamurthi, L. (2012). Demand dynamics in the seasonal goods industry: An empirical analysis. Marketing Science, 31(2), 293-316. doi: 10.1287/mksc.1110.0693

Stahlberg, M., \& Maila, V. (2010). Shopper Marketing: How to influence Consumer Decision Making at the Point of Purchase. United Kingdom: Kogan Page.

Stoll, M., Baecke, S., \& Kenning, P. (2008). What they see is what they get? An fMRI-study on neural correlates of attractive packaging. Journal of Consumer Behaviour: An International Research Review, 7(4-5), 342-359. doi: 10.1002/cb.256

Tifferet, S., \& Herstein, R. (2012). Gender differences in brand commitment, impulse buying, and hedonic consumption. Journal of Product \& Brand Management, 21(3), 176-182. doi: $10.1108 / 10610421211228793$

Triantafillidou, A., Siomkos, G., \& Papafilippaki, E. (2017). The effects of retail store characteristics on in-store leisure shopping experience. International Journal of Retail \& Distribution Management, 45(10), 1034-1060. doi: 10.1108/IJRDM-07-2016-0121

Turley, L. W., \& Milliman, R. E. (2000). Atmospheric effects on shopping behavior: A review of the experimental evidence. Journal of business research,49(2), 193-211. doi: 10.1016/S01482963(99)00010-7

Valor Investe. Valor (2019). Natal de 2019 teve maior contratação de temporários em 6 anos, diz CNC. Recuperado de https://valorinveste.globo.com/objetivo/gastarbem/noticia/2019/10/14/natal-de-2019-teve-ter-maior-contratao-de-temporrios-em-6-anosdiz-cnc.ghtml

Wilkinson, J. B., Mason, J. B., \& Paksoy, C. H. (1982). Assessing the impact of short-term supermarket strategy variables.Journal of Marketing Research,19(1), 72-86. doi: $10.1177 / 002224378201900107$

Woodside, A. G., \& Waddle, G. L. (1975). Sales Effects of In-Store Advertising. Journal of Advertising Research, 15(3), 29-34. Recuperado de: https://www.researchgate.net/profile/Arch_Woodside/publication/285455318_Sales_effects_o f_in-store_advertising/links/5eb00ca4a6fdcc7050a87d40/Sales-effects-of-in-storeadvertising.pdf

Yim, M. Y. C., Yoo, S. C., Sauer, P. L., \& Seo, J. H. (2014). Hedonic shopping motivation and co-shopper influence on utilitarian grocery shopping in superstores. Journal of the Academy of Marketing Science, 42(5), 528-544. doi: 10.1007/s11747-013-0357-2

\footnotetext{
${ }^{1}$ Layout é uma divisão da área de vendas, espaço utilizado e disposição dos produtos (Banat \& Wandebori, 2012)
} 